\title{
Visual Appearance of Chiral Nematic Cellulose-Based Photonic Films: Angular and Polarization Independent Color Response with a Twist
}

\author{
Chun Lam Clement Chan, Mélanie M. Bay, Gianni Jacucci, Roberto Vadrucci, \\ Cyan A. Williams, Gea T. van de Kerkhof, Richard M. Parker, Kevin Vynck, \\ Bruno Frka-Petesic, and Silvia Vignolini**
}

Hydroxypropyl cellulose (HPC) is a biocompatible cellulose derivative capable of self-assembling into a lyotropic chiral nematic phase in aqueous solution. This liquid crystalline phase reflects right-handed circular polarized light of a specific color as a function of the HPC weight fraction. Here, it is demonstrated that, by introducing a crosslinking agent, it is possible to drastically alter the visual appearance of the HPC mesophase in terms of the reflected color, the scattering distribution, and the polarization response, resulting in an exceptional matte appearance in solid-state films. By exploiting the interplay between order and disorder, a robust and simple methodology toward the preparation of polarization and angular independent color is developed, which constitutes an important step toward the development of real-world photonic colorants.

nematic phase capable of reflecting righthanded circularly polarized (RCP) light in the visible wavelength range. ${ }^{[5]}$ The chiral nematic pitch defines the cholesteric periodicity and consequently the reflected color, and can be tuned by many factors, such as concentration, ${ }^{[6]}$ temperature, ${ }^{[7]}$ solvent system, ${ }^{[5,8]}$ and added electrolyte. ${ }^{[9]}$

While HPC is an attractive material for responsive and low-cost photonic materials, ${ }^{[10-12]}$ the angular ${ }^{[13]}$ and polarization ${ }^{[5]}$ dependence of its optical response limits its application as a pigment. Furthermore, since the reflected color is only in the visible range when solvated $(50-70 \mathrm{wt} \%$ of HPC in water), drying results in a blueshift

Hydroxypropyl cellulose (HPC) is a biocompatible and edible cellulose derivative widely exploited in industries ranging from coatings in pharmaceutical products to various food applications, acting as binding, film-forming, or thickening agents. ${ }^{[1]}$ As a starting material for functional systems, it holds a crucial advantage over native cellulose as it is soluble in a wide range of solvents, including water, ${ }^{[2]}$ ethanol, ${ }^{[3]}$ and dimethyl sulfoxide. ${ }^{[4]}$ Aqueous HPC mesophases display lyotropic and thermotropic liquid crystalline behavior, forming a right-handed chiral

C. L. C. Chan, M. M. Bay, G. Jacucci, Dr. R. Vadrucci, C. A. Williams,

G. T. van de Kerkhof, Dr. R. M. Parker, Dr. B. Frka-Petesic, Dr. S. Vignolini Department of Chemistry

University of Cambridge

Lensfield Road, Cambridge CB2 1EW, UK

E-mail:sv319@cam.ac.uk

Dr. K. Vynck

Laboratoire Photonique

Numérique et Nanosciences (LP2N)

CNRS

IOGS

Univ. Bordeaux

33400, Talence, France

The ORCID identification number(s) for the author(s) of this article can be found under https://doi.org/10.1002/adma.201905151.

(C) 2019 The Authors. Published by WILEY-VCH Verlag GmbH \& Co. KGaA, Weinheim. This is an open access article under the terms of the Creative Commons Attribution License, which permits use, distribution and reproduction in any medium, provided the original work is properly cited.

DOI: 10.1002/adma.201905151 into a colorless state, ${ }^{[6]}$ which complicates the material processability into films or coatings. To overcome these issues, several strategies have been developed to retain color in the solid state, ranging from kinetic trapping, ${ }^{[14,15]}$ to covalent crosslinking using, e.g., diisocyanates, ${ }^{[16-18]}$ diacrylates, ${ }^{[19]}$ orthosilicates, ${ }^{[20]}$ and dialdehydes, ${ }^{[21]}$ in particular, glutaraldehyde (GA). ${ }^{[22]}$

Here we demonstrate that by exploiting the interplay between thermodynamic and kinetic effects during the selfassembly and crosslinking processes, we can tailor the desired photonic response of the HPC photonic films. Using GA as a model crosslinker, we control the level of order and disorder in the mesophase both in terms of scattering response (from the signal of individual chiral nematic domains convoluted by their orientational distribution within the mesophase) and polarization (due to the pitch deformation in highly tilted domains as well as phase retardation of the reflected signal by such domains). This combination allows for the production of macroscopically angular-independent photonic films that reflect both RCP and left-handed circularly polarized (LCP) light.

Crosslinked HPC photonic films were prepared by dissolving powder HPC into an acidic solution of glutaraldehyde such that the total concentration of HPC in solution was fixed to $60 \mathrm{wt} \%$ (see the "Experimental Section"). Under these conditions, HPC spontaneously self-assembles within minutes to form a mesophase with chiral nematic ordering. At this HPC concentration, the mesophase reflects a vibrant, iridescent "red" color, which only varies slightly with the proportion of GA crosslinker present while in solution (Figure S1, Supporting Information). Colored films were obtained from the equilibrated mesophase 

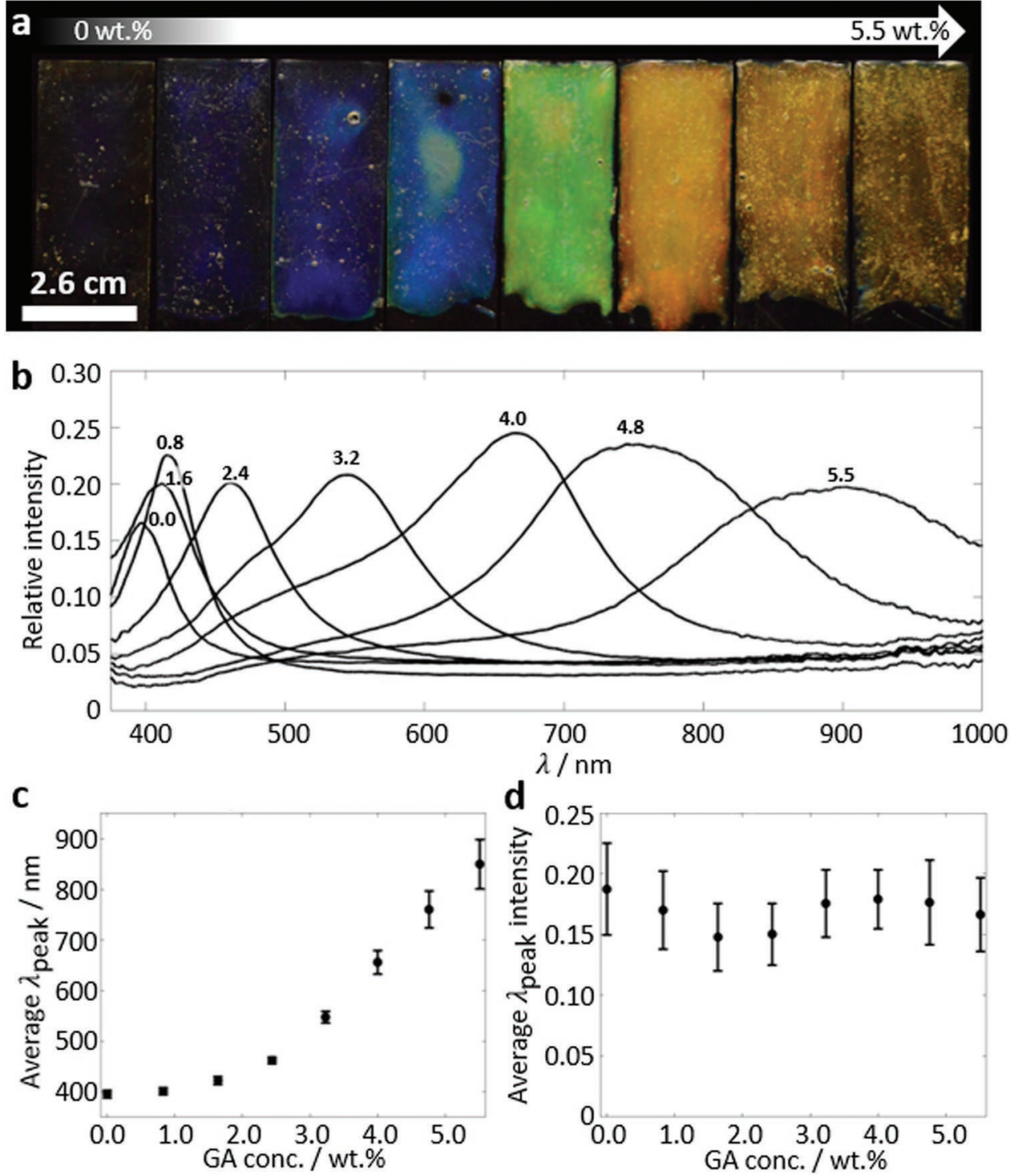

Figure 1. a) Photograph and b) dark-field reflectance spectra of HPC-GA films, demonstrating a strong redshift upon increasing the proportion of GA crosslinker. c,d) The trend in the average position (c) and intensity (d) of the peak wavelength $\left(\lambda_{\text {peak }}\right)$ plotted as a function of GA concentration in the solid film. Each point corresponds to the average of the peak wavelength or intensity of 30 dark-field spectra per composition.

via blade coating and fast solvent removal by heating at $70{ }^{\circ} \mathrm{C}$ for $2 \mathrm{~h}$. As shown in Figure 1a, the reflected color of the crosslinked HPC films can be tuned from the violet to the infrared spectral region by increasing GA concentration from 0.0 to $5.5 \mathrm{wt} \%$ in agreement with previous studies. ${ }^{[22]}$ In the absence of GA (leftmost sample in Figure 1a), the heat-treated film reflects a weak violet color whereas an analogous film will reflect in the ultraviolet if water is allowed to evaporate at room temperature. This control sample confirms that while the thermotropic behavior of HPC would redshift the resultant dried film, ${ }^{[7]}$ this effect alone is not sufficient to produce a vibrant spectrum of colored films.

During the evaporation process, from mesophase to solid film, several factors should be considered. The first is the thermodynamic equilibrium state; this depends on the temperature and on the mesophase composition, e.g., HPC concentration and ionic strength, which dynamically change during solvent evaporation. The second is the crosslinking dynamics: GA and GA-derived species ${ }^{[23]}$ reversibly form acetal or hemiacetal bonds with the hydroxyl groups in polyols, ${ }^{[24,25]}$ such as HPC, binding the polymer chains with flexible, but short linkages. Given that acetal formation would occur with a concurrent loss of water, crosslinking is also likely to be driven by the loss of solvent. As crosslinking density is increased, the ability of the HPC molecules to reach the thermodynamic equilibrium becomes constrained, especially when HPC and GA concentrations are high. Finally, the other important factor in the visual appearance is the onset of the kinetic arrest, which is the result of the interplay between the aforementioned factors and the viscosity of the solution, which increases with the proportion of GA crosslinker (Figure S2, Supporting Information). 


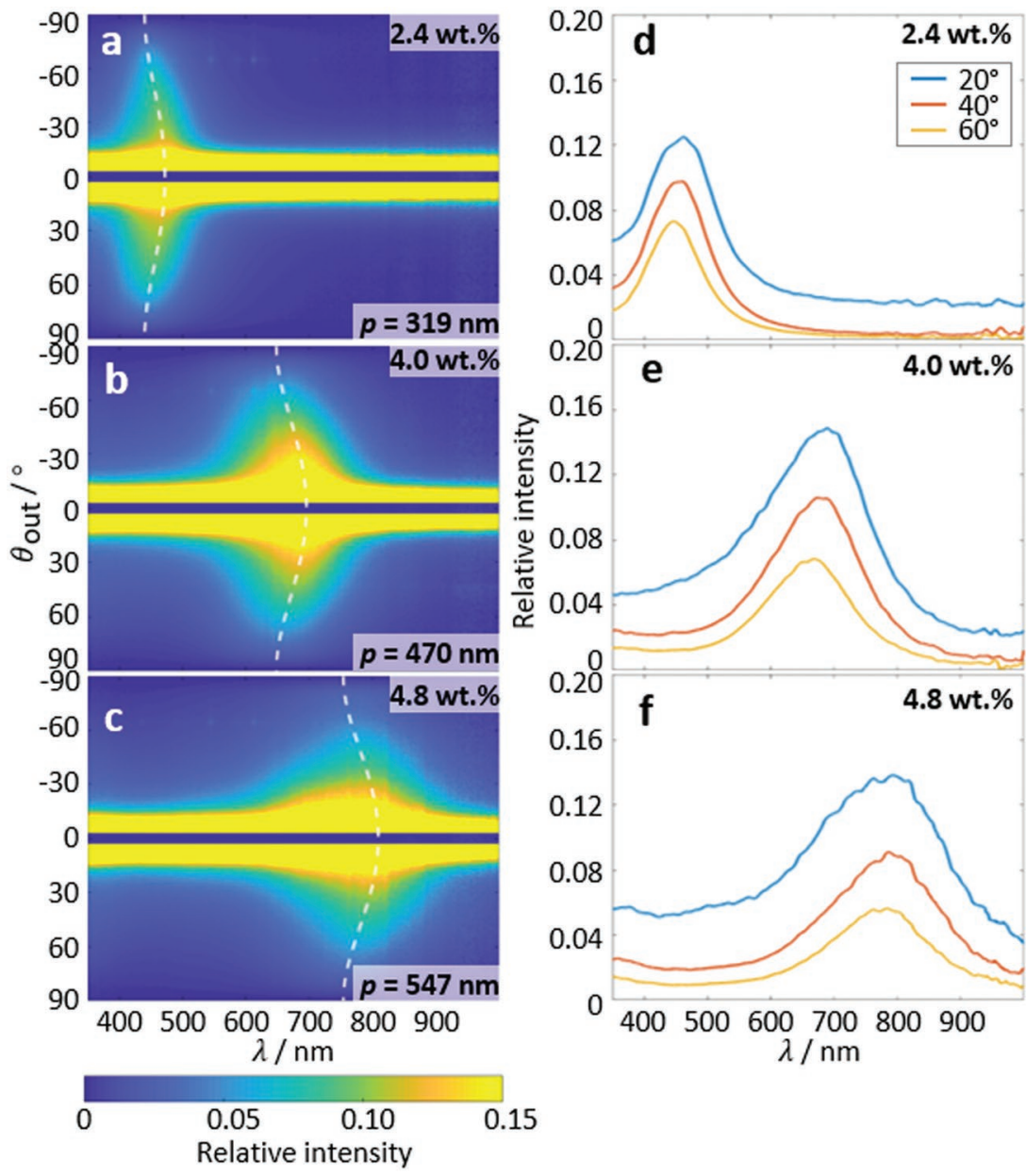

Figure 2. a-c) Intensity plots of reflectance spectra recorded as a function of observation angle for HPC-GA films at three GA concentrations. The detector was rotated around the short axis of the films. The peak wavelengths at each angle are modeled via Fergason's equation (white dashed line), allowing for the average pitch of the film to be calculated. $d-f$ ) Reflectance spectra recorded at three observation angles, as extracted respectively from (a)-(c).

By focusing only on the position of the reflected peak wavelength $\left(\lambda_{\text {peak }}\right)$, the relationship between GA concentration and the solid-state color can be divided into two regimes: a lowloading phase in which $\lambda_{\text {peak }}$ is relatively constant and a highloading one where $\lambda_{\text {peak }}$ increases approximately linearly with GA concentration (Figure 1b,c). Given the high reactivity of aldehydes and the abundance of hydroxyl groups, it is unlikely that the dialdehyde simply acts as an intercalator between the HPC layers. This is further evidenced by the lack of a residual carbonyl peak in the Fourier-transform infrared spectroscopy (FT-IR) spectrum ${ }^{[25]}$ of the HPC-GA films (Figure S3, Supporting Information). As a result, the expansion of the pitch observed in Figure 1b is likely due to the way the presence of glutaraldehyde linkers alters the packing efficiency of the HPC mesophase. At low GA concentrations, glutaraldehyde will only disrupt the packing of HPC chains locally, but macroscopically, the overall arrangement efficiently accommodates the defects and only a small variation of $\lambda_{\text {peak }}$ can be observed; so, in this region, the onset of the kinetic arrest remains mainly dominated by the self-assembly dynamics. At higher GA loading, the degree to which the structure can rearrange is limited, especially given the viscosity increases with GA content, and therefore the kinetic arrest is strongly affected by the crosslinking dynamics leading to a much larger redshift of the reflection peak. This mechanism also supports an increase in the number of packing defects with increasing crosslinking density. An increase in the disorder of the mesophase is evidenced by the spectral width of the reflected peak, which widens with increasing GA content (Figure 1b; Figures S4 and S5, Supporting Information). However, the broadening of the spectra is not accompanied by a reduction in the intensity of $\lambda_{\text {peak }}$ (Figure $1 \mathrm{~d}$ ).

Greater disorder endows the HPC-GA film with a matte appearance, which can be quantified by angular-resolved optical spectroscopy, acquired using a laboratory-built goniometer (Figure S6, Supporting Information). Figure $2 \mathrm{a}-\mathrm{c}$ shows the intensity of scattered light from three crosslinked HPC samples at different wavelengths and observation angles. At low GA loading, the sharp intensity plot indicates a uniform pitch across the sample. As the proportion of GA increases, both a loss of color purity and a larger variance in the pitch are observed. The measurements made at high GA concentration are also increasingly more difficult to fit with Fergason's equation, ${ }^{[13]}$ which predicts the angular dependence of disordered Bragg-like scattering (Figure 2a-c, white line; Figure S7, Supporting Information). This is attributed to the growing restriction to rearrangement with increasing crosslinking density that results in greater local disorder and, as such, a broader distribution of pitches between domains. Such an increase in the pitch distribution can be shown by examining the spectra of individual observation angles $\theta_{\text {out }}$. For a given $\theta_{\text {out }}$ (Figure $2 \mathrm{~d}-\mathrm{f}$ ), the convolution of signals from domains with a corresponding tilt is collected, and the spectral width increase with GA concentration is indicative of a larger multiplicity of pitches. Similar behavior has been reported in the case of another solid-state photonic system, in which an increase in the molecular weight of self-assembled block copolymers leads to redshifted structures with a concurrent loss of color purity. ${ }^{[26]}$

Once the kinetic arrest has occurred, the evaporation dynamics become important. ${ }^{[27,28]}$ For thick films, the drying rate is not homogeneous along the film profile. The loss of water occurs faster at the surface, and a "skin" is quickly formed that acts as a water barrier, slowing down the kinetics of evaporation. The rate of drying decreases significantly away from the surface, resulting in potentially two different kinetic regimes: a rapidly dried surface layer and a deeper encapsulated part that dries much slower and is observable only when the depth of the mesophase is sufficiently large (corresponding 
to a dried thickness of $\approx 500 \mu \mathrm{m}$ from Figure S8 in the Supporting Information). This difference in drying kinetics was observed in a subset of the produced films, and manifested as a redshifted spot in the middle of the film (Figures S8 and S9, Supporting Information). This redshift corresponds to domains that have been kinetically trapped at later stages of the evaporation. The difference in the pitch distribution near the surface and the bottom of the film was confirmed via scanning electron microscopy (SEM; Figure S10, Supporting Information).

The visual appearance of the HPCGA films is very different to conventional ordered chiral nematic phases of solutionstate HPC or HPC-GA (Figure S11, Supporting Information). Whereas solution-state HPC-GA appears metallic and strongly angular dependent, ${ }^{[5,29]}$ the HPC-GA composite appears matte after the drying process, as imaged in Figure 3a and in Video S1 (Supporting Information). This matte appearance is due to the fact that regardless of the viewing angle, there are always chiral nematic domains aligned favorably to reflect toward the observer, lending more angular independence to the film coloration under diffuse illumination. The diffuse character of the film's scattering response can be quantitatively described by angular-resolved scattering goniometer measurements, which were collected from the region indicated in Figure 3a. Under normal incidence, the reflected intensity at $530 \mathrm{~nm}$ measured at different observation angles can be fitted remarkably well with a Lambertian distribution (Figure 3b), and the close match between the Lambertian model and the experimental data extends over a large wavelength range (Figure S12, Supporting Information). As such, the reflective properties of the solid-state HPC film are remarkably similar to that of a perfect diffuser, which contributes to its angular-independent appearance.

While the presence of a crosslinker can increase the disorder in the HPC-GA film, the evaporation kinetics, which depends on the sample geometry, can also be responsible for the prevalence of titled domains. This can be explained by considering the mismatch between the overall thickness and the thermodynamic pitch of the mesophase. As the mesophase dries, the thickness $t$ is inversely proportional to the HPC concentration $c$ :

$t \propto c^{-1}$

However, the reduction in the thermodynamic pitch $p$ of the mesophase scales much faster with the concentration, following a power-law exponent reported to be between -2.4 and $-4.0::^{[30]}$

$p \propto c^{-n}, 2.4<n<4.0$

This mismatch effectively leads to a tensile stress on the helicoids, subsequently resulting in a Helfrich-Hurault instability and the buckling of the chiral nematic domains. ${ }^{[31,32]}$ As a result, periodic buckling can be observed in cross-sectional SEMs along both axes of the sample (Figure 3d; Figure S13, Supporting Information). Given that the periodicity of such buckling depends on the deformation experienced by the mesophase, ${ }^{[32]}$ the buckling will change over the course of the drying process and, as such, the periodicity of the resultant structure cannot be strictly defined (Sections S2 and S3 and Figure S14, Supporting Information). Optically, the buckled domains manifest as colored bands under the microscope, as seen in Figure 3c, but these bands are only evident parallel to the coating direction (i.e., the long axis). The deviation from the periodic square pattern predicted for this type of strain-induced instability ${ }^{[33]}$ is likely, especially within the bulk of the films, to be a result of shear alignment during the coating process (Figure S15, Supporting Information). Further discussion of the angular optical behavior of HPC-GA films is provided in Section S4 and Figures S16-S19 of the Supporting Information.

For aqueous HPC, the helicoidal twist of the chiral nematic phase is right-handed, and in normal incidence, only RCP light is reflected. ${ }^{[5,34]}$ In contrast, HPC-GA films reflect a large amount of LCP light over all observation angles (Figure 4a,b; Video S2, Supporting Information). To quantify the relative quantity of RCP versus LCP, we can define a degree of circular polarization (DCP), which reflects the difference between the 

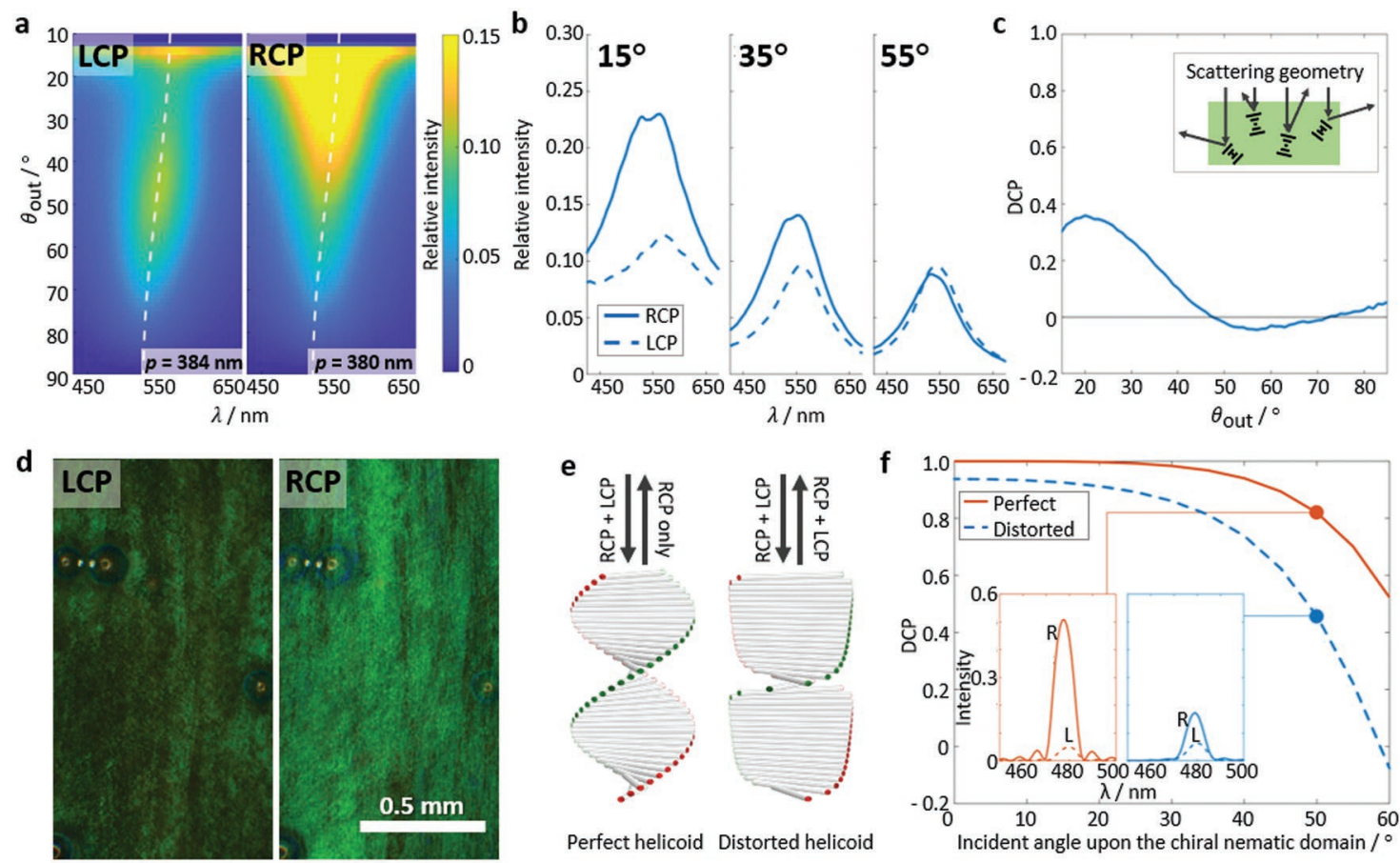

Figure 4. Characterization of a green HPC-GA film (3.2 wt\% GA). a) Intensity plot of RCP and LCP spectra recorded as a function of observation angle and with the peak wavelength at each angle modeled via Fergason's equation (white dashed line). The average pitch is calculated using the fitted equation from the Fergason model. The detector was rotated around the long axis of the sample. b) Comparison of the RCP and LCP spectral intensity at increasing observation angle. c) Plot of the measured degree of circular polarization (DCP) as a function of observation angle, with a schematic of the scattering geometry. d) Dark-field microscopy image collected in both RCP and LCP. e) Schematic of the circular polarization-dependent reflection from perfect and distorted helicoids. f) Predicted degree of circular polarization as a function of incident angle upon reflecting light from a perfect (solid line) or distorted (dashed line) chiral nematic domain, as derived using the Berreman model. Simulated RCP and LCP reflection spectra at an incident angle of $50^{\circ}$ are shown in the inset.

intensities at the peak wavelength $\left(I_{\mathrm{RCP}}\right.$ and $\left.I_{\mathrm{LCP}}\right)$ normalized by the total intensity

$\mathrm{DCP}=\frac{I_{\mathrm{RCP}}-I_{\mathrm{LCP}}}{I_{\mathrm{RCP}}+I_{\mathrm{LCP}}}$

Following this definition, an ideal right-handed helix in normal incidence will reflect exclusively RCP, corresponding to a DCP of 1, while at high angles of incidence, it will reflect a small amount of LCP light. ${ }^{[35]}$ Indeed, when the angularresolved reflection spectra of a domain consisting of a perfect right-handed helicoid are simulated via the Berreman model (methodology described in detail in Section S5 of the Supporting Information), ${ }^{[36]}$ DCP $>0.5$ across all incident angles is predicted (Figure 4f, red solid line; Figure S20, Supporting Information). Furthermore, considering that even at high sample observation angles, only reflections from relatively small incident angles can be accessed within the sample, it is expected that the relative intensity of the LCP reflection should always be negligible (Figure S20, Supporting Information). However, for HPC-GA films, at small $\theta_{\text {out }}$, DCP is $<0.4$ and trends toward 0 as $\theta_{\text {out }}$ increases, corresponding to equal amounts of LCP and RCP reflected (Figure 4c). Intriguingly, as shown in Figure 4b, a small shift can be seen between the position of the left- and right-handed signal, especially at small incident angles. While such a gap has not been explicitly described in the literature, it can be inferred from previous studies of chiral nematic structures illuminated with linear polarized light at oblique angles. When the polarizer-analyzer geometry is shifted from $s-s$ to $p-p$, the peak position was reported to shift ${ }^{[37]}$ and the difference can be shown to be dependent on the angle of the incident wave vector. ${ }^{[34]}$

Light reflected at high $\theta_{\text {out }}$ originates from highly tilted domains (see the inset in Figure 4c) and as such suggests that such domains contribute to the LCP signal while the RCP signal originates from domains more aligned to the surface. The spatial distribution of the tilted domains, which contribute to the aforementioned banded coloration, can thus be imaged via circular-polarized dark-field microscopy, where they appear as bright bands within the LCP microscopy images (Figure 4d). Such investigation demonstrates that the LCP signal can be attributed to a distortion of the chiral nematic helix, ${ }^{[27]}$ as previously reported for other chiral nematic systems. ${ }^{[38,39]}$

We explain the distortion of the chiral nematic helix as a result of the kinetic trapping of the stresses on the film during the crosslinking and drying processes. Berreman simulation of a domain with a distorted helix schematically represented in Figure $4 \mathrm{e}$ predicts that a larger amount of LCP light is reflected at larger angles of incidence (Figure 4f, blue dashed line). While in a perfectly ordered sample, such angles cannot easily be reached, in the case of the multidomain HPC-GA film, higher angles of incidence can be easily accessed due to 
multiple scattering within the mesophase (Figure S21, Supporting Information). Moreover, such highly tilted domains tend to exhibit smaller DCP not only due to the geometry of the illumination, but given buckled domains would experience a higher shear force, the distortion is likely larger for these domains. Additionally, tilted and distorted domains are linearly birefringent and can exhibit phase retardation effects (Section S6 and Figures S22-24, Supporting Information). As such, reflected RCP signal can be partially converted to LCP on transmission through such a domain. ${ }^{[40]}$ In combination, these effects lead to the relatively large amounts of LCP observed.

In conclusion, we carefully disentangled the interplay between order and disorder in solid-state cellulose-based films. We showed that solid HPC-GA films with reflection peak wavelengths across the entire visible spectrum can easily be produced through small variations in the amount of crosslinker added. We observed that the purity of the observed color at high GA content is limited by a broad spectral width. We demonstrated that the produced films possess drastically different optical behavior compared to that of solution-state HPC, showing a buckled domain structure that results in a matte and noniridescent appearance. These two characteristics are highly demanded but often lacking in dye-free, "structural pigments." Furthermore, both LCP and RCP polarizations are reflected due to distortions of the helicoidal domains, and its Lambertian character ensures that the scattered color is maximized even at low angles of incidence. Engineering a colorful solid material from such a highly concentrated solution of liquid crystalline polymer puts unusual constraints on the crosslinking possibilities to maintain and control the chiral nematic behavior of the initial solution. Further developments by employing more biocompatible crosslinkers and enhancing color appearance via the inclusion of black absorbents will certainly benefit the development of crosslinked HPC as photonic pigments for a variety of applications, including cosmetics and food additives. In that context, this strategy for producing a low-cost and structurally colored material from HPC and retaining both angular independency and low polarization dependence constitutes an important step toward the development of these products.

\section{Experimental Section}

Sample Preparation: Dry hydroxypropyl cellulose powder (HPC SSL SFP, food grade, $M_{\mathrm{w}} \approx 40000 \mathrm{~g} \mathrm{~mol}^{-1}$ as reported by manufacturer) was obtained from Nisso Chemical Europe. Glutaraldehyde (50 wt $\%$ aqueous solution) was purchased from Alfa Aesar. All materials were used as supplied and without further purification.

Solvated HPC-glutaraldehyde mixtures with a starting concentration of $2.0 \mathrm{wt} \% \mathrm{GA}$ and $60 \mathrm{wt} \%$ HPC (i.e., $3.2 \mathrm{wt} \% \mathrm{GA}$ in the final product) were prepared by first diluting glutaraldehyde $(0.4 \mathrm{~g})$ in aqueous $\mathrm{HCl}$ $(0.5 \mathrm{~m}, 3.6 \mathrm{~g})$. This acidic glutaraldehyde solution was subsequently added to dry HPC $(6.0 \mathrm{~g})$, and immediately mixed using a planetary centrifuge (Thinky ARE-250, with THI150ML container \& THI250AD-201 adaptor), followed by degassing via centrifugation at $5000 \times \mathrm{g}$ for $30 \mathrm{~min}$ (Thermofisher Sorvall LYNX 6000, with T29 rotor). Samples with differing amounts of GA were prepared following the same procedure. The mass of HPC and glutaraldehyde used corresponded to their respective weight percentage while the remaining weight percentage equated to the amount of dilute $\mathrm{HCl}$ added. Table S1 (Supporting Information) relates the final concentration of GA (relative to the dry HPC-GA film) to the starting concentration of GA (relative to the HPC mesophase).
In addition, it should be noted that the $\mathrm{pH}$ was not standardized to account for the small amount of water included with the glutaraldehyde.

Film Preparation: HCP-GA films were coated onto a glass slide $(1.1 \times$ $2.6 \times 7.6 \mathrm{~mm}$ ) via a custom-made blade-coating setup, with a gap of $2.0 \mathrm{~mm}$ (an effective gap of $0.9 \mathrm{~mm}$ after accounting for the thickness of the glass slide) and a speed of $0.5 \mathrm{~mm} \mathrm{~s}^{-1}$ (Figure S25, Supporting Information). The films were then transferred into a furnace oven at room temperature and heated up to $70^{\circ} \mathrm{C}$ over $5 \mathrm{~min}$. The samples were then held at $70{ }^{\circ} \mathrm{C}$ for $2 \mathrm{~h}$ at which point the dry films were removed from the oven and allowed to cool to room temperature (during which no further color change was observed).

Characterization: Dark-field microscopy was carried out using a Zeiss Scope.A1 microscope at $5 \times$ magnification (Zeiss EC Epiplan-NEOFLUAR $5 \times / 0.13$ HD DIC objective). To record reflectance spectra, the microscope was coupled to a spectrometer (Avantes AvaSpec-HS2048), with a $600 \mu \mathrm{m}$ core optical fiber (Thorlabs FC-UV600). All spectra were measured relative to a standard white diffuser (Labsphere SRS-99-010). Circular polarized optical microscopy was performed using an analyzer composed of a linear polarizer and a quarter-wave plate with its slow axis oriented either at $+45^{\circ}$ or $-45^{\circ}$ to selectively probe RCP or LCP. On each film, of which three were prepared per GA concentration, spectra were recorded at ten locations and the reported $\lambda_{\text {peak }}$ for each composition was an average of all 30 measurements while the error was taken as the standard deviation of these measurements. Measurements in a bright field geometry were not collected as they were dominated by the specular reflection of the film surface. Stereomicroscopy was carried out using a Zeiss Stemi 305 microscope.

Angular-resolved optical spectroscopy measurements were performed using the setup described in detail in Figure S19 (Supporting Information). To summarize, the sample was illuminated by a collimated light source (Ocean Optics HPX-2000 xenon lamp) at a normal incidence $\left(\theta_{\text {in }}=0^{\circ}\right)$. The scattered light was collected as a function of angles, $\theta_{\text {out }}$, using a moving arm to scan either between $-90^{\circ}<\theta_{\text {out }}<90^{\circ}$ or $0^{\circ}<\theta_{\text {out }}<90^{\circ}$. Unless stated otherwise, a spectrum was recorded at $1^{\circ}$ increments. To examine the polarization of the scattered light, linear polarizer and quarter-wave plates were introduced when required. The data obtained were fitted using Fergason's equation, ${ }^{[13]}$ assuming an average refractive index of 1.49 for all samples. ${ }^{[41]}$

Scanning electron microscopy was conducted on a TESCAN MIRA3 FEG-SEM system. Cross-sectional samples were mounted in between two aluminum plates and sputter-coated with $\mathrm{Pt}(10 \mathrm{~nm})$ prior to measurements (Quorum Technologies Q150T ES).

\section{Supporting Information}

Supporting Information is available from the Wiley Online Library or from the author.

\section{Acknowledgements}

This work was funded by EPSRC grant EP/R511675/1 to B.F.-P. and S.V.; by EPSRC grant EP/L016087/1 to C.A.W.; by BBSRC David Phillips fellowship BB/K014617/1 to G.J. and S.V.; by EU's Horizon 2020 research and innovation programme under the Marie Skodowska-Curie grant agreement No. 722842 (ITN Plant-inspired Materials and SurfacesPlaMatSu) to G.T.v.d.K. and S.V.; by Swiss National Science Foundation \#165176 to R.V.; by Croucher Cambridge International Scholarship to C.L.C.C.; by ERC grant ERC-2014-STG H2020 639088 to M.M.B., G.J., and S.V.; by ERC grant ERC-2017-POC 790518 to R.M.P. and S.V. C.L.C.C. thanks Dr. Renata L. Sala for guidance on the rheological measurement. Additional data relating to this publication is available from the University of Cambridge data repository (https://doi.org/10.17863/CAM.45745).

\section{Conflict of Interest}

The authors declare no conflict of interest. 


\section{Keywords}

chiral nematic liquid crystals, hydroxypropyl cellulose, structural color

Received: August 9, 2019

Revised: September 25, 2019

Published online: November 18, 2019

[1] Klucel Hydroxypropylcellulose Physical and Chemical Properties (Datasheet), Ashland Inc., 2017.

[2] R. S. Werbowyj, D. G. Gray, Mol. Cryst. Liq. Cryst. 1976, 34, 97.

[3] S. A. Vshivkov, L. V. Adamova, E. V. Rusinova, A. P. Safronov, V. E. Dreval, A. G. Galyas, Polym. Sci., Ser. A 2007, 49, 578.

[4] S. Suto, H. Tashiro, M. Karasawa, J. Appl. Polym. Sci. 1992, 45, 1569.

[5] R. S. Werbowyj, D. G. Gray, Macromolecules 1984, 17, 1512.

[6] R. S. Werbowyj, D. G. Gray, Macromolecules 1980, 13, 69.

[7] S. Fortin, G. Charlet, Macromolecules 1989, 22, 2286.

[8] G. Conio, E. Bianchi, A. Ciferri, A. Tealdi, M. A. Aden, Macromolecules 1983, 16, 1264.

[9] Y. Nishio, R. Chiba, Y. Miyashita, K. Oshima, T. Miyajima, N. Kimura, H. Suzuki, Polym. J. 2002, 34, 149.

[10] H. Liang, M. M. Bay, R. Vadrucci, C. H. Barty-King, J. Peng, J. I. Baumberg, M. F. L. De Volder, S. Vignolini, Nat. Commun. 2018, 9, 4632.

[11] G. Kamita, B. Frka-Petesic, A. Allard, M. Dargaud, K. King, A. G. Dumanli, S. Vignolini, Adv. Opt. Mater. 2016, 4, 1950.

[12] A. Espinha, C. Dore, C. Matricardi, M. I. Alonso, A. R. Goñi, A. Mihi, Nat. Photonics 2018, 12, 343.

[13] J. L. Fergason, Mol. Cryst. 1966, 1, 293.

[14] A. Tanaka, H. Onoda, K. H. Nitta, Polym. J. 2000, 32, 665.

[15] G. Charlet, D. G. Gray, Macromolecules 1987, 20, 33

[16] P. Almeida, S. Tavares, A. Martins, M. Godinho, M. Cidade, J. Figueirinhas, Opt. Mater. 2002, 20, 97.

[17] T. G. Rials, W. G. Glasser, J. Appl. Polym. Sci. 1988, 36, 749.

[18] S. Suto, H. Tashiro, M. Karasawa, J. Mater. Sci. Lett. 1990, 9, 768.
[19] R. Chiba, Y. Nishio, Y. Sato, M. Ohtaki, Y. Miyashita, Biomacromolecules 2006, 7, 3076.

[20] A. Thomas, M. Antonietti, Adv. Funct. Mater. 2003, 13, 763.

[21] S. Suto, N. Ui, J. Appl. Polym. Sci. 1996, 61, 2273

[22] S. Suto, S. Hasegawa, J. Mater. Sci. 2002, 37, 4857.

[23] I. Migneault, C. Dartiguenave, M. J. Bertrand, K. C. Waldron, BioTechniques 2004, 37, 790.

[24] K. Tomihata, Y. Ikada, J. Polym. Sci., Part A: Polym. Chem. 1997, 35, 3553.

[25] H. S. Mansur, C. M. Sadahira, A. N. Souza, A. A. P. Mansur, Mater. Sci. Eng., C 2008, 28, 539.

[26] D. P. Song, G. Jacucci, F. Dundar, A. Naik, H. F. Fei, S. Vignolini, J. J. Watkins, Macromolecules 2018, 51, 2395.

[27] B. Frka-Petesic, G. Kamita, G. Guidetti, S. Vignolini, Phys. Rev. Mater. 2019, 3, 045601

[28] R. M. Parker, G. Guidetti, C. A. Williams, T. Zhao, A. Narkevicius, S. Vignolini, B. Frka-Petesic, Adv. Mater. 2018, 30, 1704477.

[29] H. de Vries, Acta Crystallogr. 1951, 4, 219.

[30] M. H. Godinho, D. G. Gray, P. Pieranski, Liq. Cryst. 2017, 44, 2108.

[31] C. A. Whitfield, T. C. Adhyapak, A. Tiribocchi, G. P. Alexander, D. Marenduzzo, S. Ramaswamy, Eur. Phys. J. E: Soft Matter Biol. Phys. 2017, 40, 50.

[32] N. Scaramuzza, R. Bartolino, G. Barbero, J. Appl. Phys. 1982, 53, 8593.

[33] N. A. Clark, R. B. Meyer, Appl. Phys. Lett. 1973, 22, 493.

[34] V. A. Belyakov, V. E. Dmitrienko, V. P. Orlov, Usp. Fiz. Nauk 1979, 127, 221.

[35] E. Kuze, Y. Ouchi, M. Hara, A. Fukuda, H. Takezoe, Jpn. J. Appl. Phys. 2005, 22, 1080.

[36] D. W. Berreman, J. Opt. Soc. Am. 1972, 62, 502.

[37] D. W. Berreman, T. J. Scheffer, Mol. Cryst. Liq. Cryst. 1970, 11, 395.

[38] M. Rivera, J. A. Reyes, Appl. Phys. Lett. 2007, 90, 023513.

[39] A. J. J. Kragt, D. C. Hoekstra, S. Stallinga, D. J. Broer, A. P. H. J. Schenning, Adv. Mater. 2019, 31, 1903120.

[40] A. M. Ritcey, G. Charlet, D. G. Gray, Can. J. Chem. 1988, 66, 2229.

[41] Y. Onogi, Y. Nishijima, Kobunshi Ronbunshu 1986, 43, 223. 